\title{
Costs dictate strategic investment in dominance interactions
}

Tobit Dehnen ${ }^{1,2,3}$, Danai Papageorgiou ${ }^{2,3,4,5}$, Brendah Nyaguthii ${ }^{6,7.8}$, Wismer Cherono ${ }^{7}$, Julia Penndorf ${ }^{9}$, Neeltje J. Boogert ${ }^{1}$, Damien R. Farine $e^{2,3,7}$

${ }^{1}$ Centre for Ecology and Conservation, University of Exeter, Penryn Campus, Penryn TR10 9FE, UK

${ }^{2}$ Department of Evolutionary Biology and Environmental Studies, University of Zurich, 8057 Zürich, Switzerland

${ }^{3}$ Department of Collective Behavior, Max Planck Institute of Animal Behavior, 78464 Konstanz, Germany

${ }^{4}$ Kenya Wildlife Service, P.O. Box 40241-001000, Nairobi, Kenya

${ }^{5}$ University of Konstanz, Department of Biology, 78457 Konstanz, Germany

${ }^{6}$ Department of Ornithology, National Museums of Kenya, P.O. Box 40658-001000, Nairobi, Kenya

${ }^{7}$ Mpala Research Centre, P.O Box 555-10400, Nanyuki, 10400, Kenya

${ }^{8}$ University of Eldoret, School of Natural Resource Management, Department of Wildlife, 1125-30100 Eldoret, Kenya

${ }^{9}$ Cognitive and Cultural Ecology Research Group, Max Planck Institute for Animal Behavior, 78315 Radolfzell, Germany

\section{Author for correspondence:}

Damien R. Farine

e-mail: damien.farine@ieu.uzh.ch 


\section{Abstract}

Dominance is important for access to resources. As dominance interactions are costly, individuals should be strategic in who they interact with. One hypothesis is that individuals should direct costly interactions towards those closest in rank, as they have most to gain-in terms of attaining or maintaining dominance-from winning such interactions. Here, we develop a novel analytical framework to test whether interactions are directed strategically in relation to rank differences, and use these to compare strategies across types of interactions that vary in cost. We show that male vulturine guineafowl (Acryllium vulturinum), a gregarious species with steep dominance hierarchies, strategically direct costly interactions towards males occupying ranks immediately below themselves in their group's hierarchy. In contrast, low-cost interactions are not directed towards closest competitors, but towards group members slightly further down the hierarchy. We then show that, as a result of the difference in strategic use of high- and low-cost interactions towards closest competitors, individuals disproportionately use highest-cost interactions-such as chases-towards males found one to three ranks below them. Our results support the hypothesis that the costs associated with different interaction types can determine their expression in social groups.

Keywords: Aggression, dominance hierarchy, rank, social behaviour, social cognition, social structure. 


\section{Introduction}

In many group-living species individuals have conflicting interests over the use of limited resources [1], often resulting in aggressive interactions [2]. As individuals typically differ in their ability to win such interactions [3], differences in winning propensity give rise to group-level patterns known as dominance hierarchies. Individuals' resulting position in dominance hierarchies can have profound consequences for access to important resources such as food [4,5], preferential roosting positions [6] and reproductive opportunities [7], highlighting the importance of rising to the top of the hierarchy a challenge faced by individuals across the animal kingdom [8]. However, while dominance interactions are ultimately beneficial to some individuals, they also involve costs - such as the depletion of energy reserves $[9,10]$, the time spent engaging in dominance interactions [11], the risk of substantial injury [12] and enhanced predation risk [13]. Accordingly, one hypothesis is that, to maximise the net benefits, individuals should be strategic in terms of whom they interact with and how much to invest in dominance interactions with conspecifics, such that they may attain-and maintain -their position in the hierarchy at minimal cost to themselves [14,15]. Indeed, theoretical models suggest that directing interactions towards close competitors can stabilise a hierarchy [16]. Under such a scenario, individuals should invest more, i.e. incur greater costs, in aggressive interactions directed towards competitors closer in the hierarchy, as the directionality of the dominance relationship is likely to be most vulnerable to change between closely-matched competitors $[17,18]$. By contrast, the directionality of dominance relationships with individuals much further away in the hierarchy is unlikely to be reversed. Thus, we should expect to observe more highcost interactions than expected by chance among individuals with similar ranks, and for this pattern to weaken as interactions become less costly (e.g. low-cost aggressive or submissive interactions).

Previous work suggests that, at least in some species or groups, individuals appear to interact strategically in relation to the relative ranks of group members. For example, Wright et al. [14] found a higher frequency of aggression among male mountain gorillas (Gorilla beringei beringei) that were close in rank. Similarly, Hobson \& DeDeo [19] found that individuals in captive groups of monk parakeets (Myiopsitta monachus) direct aggression preferentially towards individuals close in the hierarchy. Alternatively, individuals could direct interactions evenly across all lower-ranked group members, or it has been suggested that 'bullying'-aggression directed preferentially toward individuals at the bottom of the hierarchy-may also be a strategy [20]. However, it remains to be established whether strategies are linked to the costs of the interaction, such that higher-cost interactions (like fights, which can lead to injury) might be expressed more strategically than low-cost interactions (such as submissive interactions, where one individual simply signals its subordinate status). Differences in the frequency and targets of different interaction categories, driven by costs, 
could mask behavioural strategies. For example, if submissive-or even low-cost aggressiveinteractions are more commonly expressed than fights, but pooled together with data on fights, it could appear as if individuals in a given group are not strategic in their interactions. Partitioning out the expression and targets of interactions involving different cost may reveal much more widespread use of interaction strategies. Thus, whether strategies vary between interaction categories is an avenue of enquiry that necessitates further research.

Inferring whether individuals express interaction strategies, and how this varies between interaction types, requires meeting several challenges. First, it requires a large dataset of interactions for each interaction category that can be split into two subsets to independently estimate individuals' position in the hierarchy as well as their potential strategies. Second, data collection should ideally be replicated across multiple social groups to determine whether the inferred strategies are commonly used (as expected if they have been under selection). Third, because groups are often socially structured [21,22], inference will require quantifying how individuals express dominance interactions relative to their opportunity to do so. For example, even in seemingly closed social groups (i.e. those with stable membership), individuals can vary in their propensity to be in the same part of the group, or subgroup, for example according to rank $[23,24]$ or age and sex [25]. Such spatial clustering could lead to some dyads (sets of two individuals) being over-represented in the interaction dataset simply because they are more often in close spatial proximity.

In this study, we start by extending existing analytical methods $[19,20]$ to infer the strategic us of dominance interactions. Specifically, we first introduce a randomised data-splitting approach to estimate the dominance hierarchy independently of the interaction strategy to ensure that these are estimated independently. Repeating this approach in a bootstrapping-like fashion has the additional advantage of providing estimates of uncertainty in the relationship inferred between interaction strategy and relative hierarchy position. We next extend current methods by including a null modelhere a permutation test [26] - to allow the observed patterns of interactions to be compared to interaction opportunity, thereby revealing whether individuals adopt interaction strategies while controlling for differences in opportunity. We then use a simple agent-based model to demonstrate the functionality of our method, including a) how apparent strategies can arise solely as a result of social structure, and b) how our permutation-based approach resolves such situations. We apply our approach to test whether males of two vulturine guineafowl (Acryllium vulturinum) social groups use high-cost interactions (e.g. chases), low-cost interactions (e.g. displacements) or submissive interactions strategically. Finally, we use Bayes' rule to explicitly test whether males disproportionately express highest-cost interactions (relative to lower-cost interactions) towards individuals nearest in rank. 


\section{Methods}

\section{A general method for inferring strategic use of dominance interactions}

We develop a general framework for inferring whether individuals express rank difference-based strategies in dominance interactions. Our method builds on the approach proposed by Hobson \& DeDeo [19], further developed by Hobson et al. [20], in that it generates an estimate of the relationship between the propensity for individuals to interact and their differences in dominance rank. We refine this approach in two important ways. The first is to introduce a data-splitting approach, to independently estimate rank differences vs. tendencies to interact, and to repeat the data-splitting approach in a bootstrapping-like process to provide an estimation of the uncertainty of the relationship. The second is to use permutation tests to quantify how the observed interaction frequency compares to the expected interaction frequency given opportunity alone. We refer to this interaction frequency corrected for opportunity as 'the tendency to interact'. Our framework consists of six steps (Figure 1).
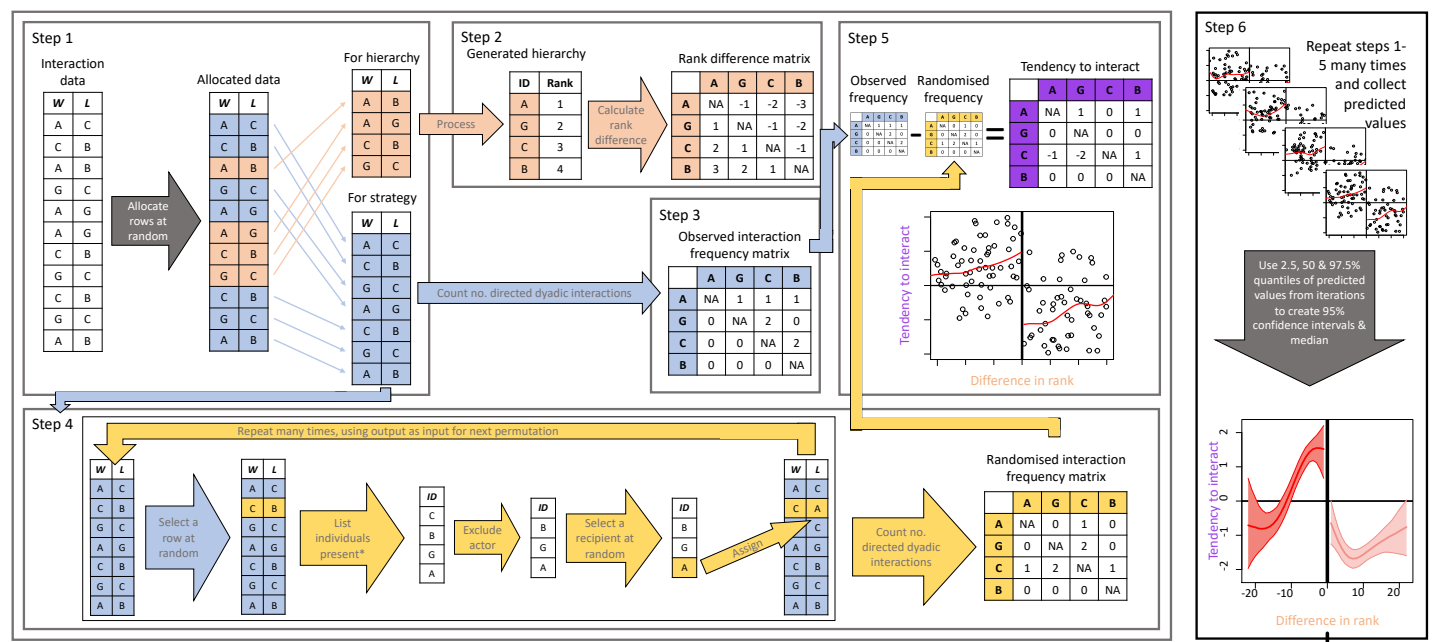

Figure 1. Overview of the proposed approach. Step 1: The dataset of winners $(\boldsymbol{W})$ and losers $(\boldsymbol{L})$ of interactions (rows) is randomly split into two subsets, one to generate a hierarchy (orange) and one to infer interaction strategies (blue). Step 2: A hierarchy is generated and differences in hierarchy position are calculated for each pairwise combination of actors and recipients (dyad). Differences, which can be either in rank or (e.g. Elo) score depending on whether it is an rank- or score-based hierarchy, are stored in a matrix where the number reflects the rank/score of the column relative to the row. Step 3: The sum of directed interactions within each dyad are counted using the 'for strategy' (blue) data, storing these in a directed matrix. Step 4: A permutation procedure-here involving the repeated random allocation of interaction recipients from a pool of possible recipients (*based on observation of local group composition at the time the interaction took place)-generates a randomised interaction frequency dataset, which is stored in a directed matrix to match the observed interaction frequency matrix. Step 5: Differences between the observed and random interaction frequencies are calculated, producing a tendency to interact matrix. The relationship between rank/score difference and tendency to interact is then modelled using a method for estimating nonlinear relationships, such as splines. Step 6: Steps 1-5 are repeated many times (e.g. 1000) by repeatedly allocating random parts of the data to each subset, and the predicted values of each model are stored. These predicted values are then used to estimate the confidence intervals at each rank difference, thereby revealing whether the tendency to interact is significantly different to the null expectation (when the range between the upper and lower confidence intervals does not overlap 0 for a given rank difference). 
The first step in our framework splits the dataset into two subsets, allowing the independent inference the dominance hierarchy (predictor) and the tendency to interact (response variable) - as is statistically preferable. Here, a randomly selected subset of the data (e.g. $30 \%$ ) is used to calculate individuals' hierarchy positions (e.g. via ranks or Elo scores) and then pairwise positional differences. The remaining (e.g. 70\%) non-overlapping portion of the dataset is used to infer pairwise interaction rates. Accordingly, the dataset is randomly split for generating the two axes of interest - differences in hierarchy position and interaction rates.

The second step is to use the hierarchy-inference subset of the data to generate a hierarchy. This can be done using a range of methods, and can generate either rank- $[27,28]$ or score-based [29] hierarchies. We suggest using the method proposed by Sánchez-Tójar et al. [30], which combines Elo scores with a temporal randomisation procedure that has been shown to increase accuracy for static hierarchies.

The third step in our framework is to count the number of directed interactions across each pairwise combination of actors and recipients (herein a dyad). We opt for this option, rather than count the number of interactions per unit of rank difference, because we naturally expect smaller rank differences to be over-represented in the data. That is, for $\mathrm{N}$ individuals, there are N-1 dyads with a rank difference of $1, \mathrm{~N}-2$ dyads with a rank difference of 2 , and so on, with only one dyad having the maximal rank difference of $\mathrm{N}-1$. Thus, simply counting how many interactions occur across all dyads for each unit of rank difference would create a positive linear relationship under the null hypothesis (no strategy). Further, maintaining these data at the dyadic level allows the use of either rank- or score-based estimations of dominance (here we refer to both these as simply ranks). This process produces a dyadic count of the number of interactions, which can be matched up with the difference in rank for that dyad. Note that the relationship is directed and asymmetric; the difference in rank is negative if the actor $A$ is higher ranked than the recipient $B$, and positive otherwise, and the number of interactions will generally be asymmetric (individual A may be the actor much more often than individual B).

The fourth step is to generate an expected pattern of interactions given opportunity. For this, we implement a permutation procedure, which requires data on the local composition of the group (or subgroup) when the interaction took place. Each iteration of the permutation runs as follows:

(1) randomly select one interaction and compile a list of all individuals present when the interaction took place, 
(2) remove the actor (aggressor or submissive individual) from this list, and

(3) randomly select an individual from the list as the new recipient of the interaction (this can include the original recipient).

The procedure is run iteratively for a predefined number of permutations (e.g. 100,000), producing a new 'random' dataset (i.e. the dataset after the final permutation).

The fifth step is to generate a measure of tendency to interact that controls for dyadic opportunity to interact. We suggest using the difference between the observed and permuted datasets, i.e. the observed interaction count for each dyad minus their expected count from the permuted dataset. Using this measure, 0 represents a tendency to interact at the expected frequency, positive values represent an actor that interacted more often with the recipient than expected by chance, and negative values represent those that interacted less often than expected by chance. This measure, estimated for each dyad, can be plotted against their difference in hierarchy position, and modelled. We suggest modelling using a method for estimating non-linear relationships, such as splines, as the sum of the tendency values is zero, and therefore a linear relationship is only expected when interactions are random or perfectly stratified across ranks.

The above process (steps 1-5) can be repeated many times, each time with different parts of the data allocated to each procedure. Thus, the sixth step in our framework is to repeatedly (e.g. 1000 times) estimate the relationship between the difference in hierarchy position and the tendency to interact, to produce an estimate of uncertainty. This can be done, for example, by calculating the $95 \%$ range of the predicted tendency to interact-values at set intervals along the difference in hierarchy positionvariable from the distribution of splines produced. For differences in hierarchy position in which the 95\% range does not overlap with 0 , the tendency to interact can be considered as being either significantly greater than expected by chance (when the entire $95 \%$ range is above 0 ) or lower than expected by chance (when the entire range is below 0 ).

\section{Testing the framework}

We demonstrate the potential pitfalls that can arise when analysing dominance datasets, and how the application of our analytical framework addresses these, using a simple agent-based model. The model starts by creating $X$ groups with a given average size $G$ drawn from a population of size $N$. Individuals can be drawn from this pool either at random, or can be assorted into subgroups by dominance. Thus, we are able to test whether our approach, when inferring tendency to interact, correctly accounts for subgrouping in scenarios where individuals either assort according to rank, as 
may occur in real animal groups [24], or are grouped randomly. In the random scenario, each individual has an equal binomial probability $P_{i}=G \times \frac{1}{N}$ of being drawn in a given group. In the assorted scenario, we first assign a target rank $z$ to each group by randomly drawing a number from 1 to $N$, and then setting a binomial probability that each individual $j$ with rank $r_{j}$ is drawn to $P_{i, j}=$ $\frac{1}{2 \sqrt{2 \pi}} e^{-\frac{\left(r_{j}-z\right)^{2}}{8}}$. This equation corresponds to the estimated probability density at $r_{j}$ in a normal distribution with a mean of $z$ and a standard deviation of 2 . We finally normalise the $P_{i, j}$ values such that they sum to $G$. For each group, we then set group membership for each individual by drawing a 1 (present) or 0 (absent) using the binomial probabilities $P_{i}$ or $P_{i, j}$, but ensuring that each group has at least two individuals.

For each group, we simulate an interaction between two individuals by first drawing from the present individuals at random. We then calculate the probability that the more dominant individual would win as $P_{\text {win }}=1-\left(1-P_{w}\right)^{r_{d}}$, where $P_{w}$ is the probability that a dominant individual wins given a rank difference of 1 , and $r_{d}$ is the absolute difference in rank (i.e. the difference in rank as a positive number). We then produce an outcome from a binomial draw with the probability of the dominant winning being given by $P_{w}$ and the probability of the subordinate winning by $\left(1-P_{w}\right)$.

We evaluate the outputs of both scenarios (random and assorted) using raw interaction counts and using our framework. We create $N=20$ individuals interacting in $X=100$ groups, split the data $70 \%$ to calculate strategies, and run 1000 permutations for each iteration of the analysis. We set $P_{w}$ to 0.9 and $G=5$.

\section{Empirical data}

Study species: We studied two habituated social groups of individually colour-banded vulturine guineafowl living in the savannah and dry-woodland habitat surrounding Mpala Research Centre $\left(0^{\circ} 17^{\prime} \mathrm{N}, 37^{\circ} 52^{\prime} \mathrm{E}\right)$ in Laikipia County, Kenya. These birds live in stable social groups [31,32] and frequently engage in aggressive and submissive interactions with group members that result in steep dominance hierarchies [33].

Data collection: We collected data between $6^{\text {th }}$ September 2019 and $10^{\text {th }}$ February 2021 . We recorded the winners and losers of eight distinct types of dyadic dominance interactions (see Table 1) using an all-occurrence sampling method [34]. Such observations typically took place while following social groups (on foot or by car) between 0600 and 0930 or 1500 and 1830 . We collected data on $76 \%$ of the 
days within the start and end dates. As not all group members are present at all times, or groups can sometimes be split into spatially distinct subgroups, we also recorded group composition information during data collection. We defined subgroups as cohesive sets of individuals that are spaced $\sim 50$ meters or more apart, but also used cues such as direction of movement and cohesiveness to define subgroups.

Table 1. Vulturine guineafowl (Acryllium vulturinum) exhibit a range of aggressive (1-6) and submissive (7-8) dyadic dominance interactions (see also Papageorgiou \& Farine [33]).

\begin{tabular}{|c|c|c|c|}
\hline & Interaction category & Interaction type & Description \\
\hline 1 & High-cost aggressive & SPI & A grabs $B$ by the tail feathers and spins them around \\
\hline 2 & High-cost aggressive & TAl & A grabs $B$ by tail or body feathers \\
\hline 3 & High-cost aggressive & $\mathrm{CHA}$ & A chases $B$ \\
\hline 4 & Low-cost aggressive & PEC & A gently pecks B on the head or on body \\
\hline 5 & Low-cost aggressive & DIS & $\begin{array}{l}\text { A displaces B from foraging or resting (e.g. sand- } \\
\text { bathing) spot }\end{array}$ \\
\hline 6 & Low-cost aggressive & GAP & $\begin{array}{l}\text { A gapes at B (similar to chase display but without } \\
\text { chasing). B recedes }\end{array}$ \\
\hline 7 & Submissive & SUV & $\begin{array}{l}\text { A falls to the ground in front of } B \text {, often } \\
\text { accompanied by a 'crying call'. Usually observed in } \\
\text { chicks, but also between adults when on the move }\end{array}$ \\
\hline 8 & Submissive & SUB & $\begin{array}{l}\text { A performs submissive caress with its body } \\
\text { under/around the chest of B }\end{array}$ \\
\hline
\end{tabular}

Interaction categories: Three co-authors (TD, DP and BN) independently arranged aggressive interactions in order of intensity (as has been done previously for other taxa [35]). Aggressive interactions were then split into two halves, as three of these interactions-namely SPI, TAI and CHAare of considerably higher intensity, and likely more costly to the actor in terms of energy and time expenditure as well as potential injury risk, than the other three - PEC, DIS, GAP (Table 1). The two submissive interactions-SUB and SUV-were deemed similarly costly, as submissive interactions typically function to signal existing dominance relationships-e.g. 'pant-grunt' vocalizations in chimpanzees (Pan troglodytes) [36,37]-and neither is especially intense. Accordingly, we categorised each dyadic dominance interaction as either i) high-cost aggressive, ii) low-cost aggressive or iii) submissive (Table 1 ).

Calculating ranks: All analyses only consider interactions that occurred within a sex, as the costs and benefits of interacting likely differ between the sexes, which may thus follow different strategies. For this study, we focus on male-male interactions because male vulturine guineafowl tend to interact more frequently. Additionally, adult males are typically dominant over all females in the group [33] and thus represent an uninterrupted hierarchy. Group one contained 23 and group two contained 29 males. In each analysis, we define rank differences as the rank of the actor minus that of the recipient. For example, if individuals $A$ and $B$ are ranked at 4 and 6 , respectively, then the tendency to interact 
for $A$ to $B$ would be plotted at rank difference $=-2$; in contrast, the tendency to interact for $B$ to $A$ would be plotted at rank difference $=2$. We also repeat our main analysis using a score-based hierarchy, by skipping the conversion of Elo scores into ranks, but normalising scores to be between 0 and 1 (as the range of raw Elo scores can differ across different runs of the simulation). Ranks and Elo scores are calculated using the aniDom R package v 0.1.4 [30].

Robustness of our analyses to methodological decisions: We first test whether hierarchies constructed using each of the three different interaction categories are equivalent. We do this by calculating the correlation between the ranks inferred from each set of interaction categories, for each social group, using the Spearman's rank correlation coefficient (hereafter $r_{s}$ ). If all three hierarchies are closely correlated, the interactions in different interaction categories can be assumed to represent the same axis of dominance, confirming that findings across different interaction categories are comparable and robust.

We then test the within-hierarchy robustness of the data by running a repeatability analysis on subsets of the data (i.e. after splitting in step 1 in Figure 1) to determine hierarchy uncertainty. First, we randomly choose $30 \%$ of interactions of a given subset, matching the proportion of interactions used for rank-inference in the main analysis. Second, we use the function estimate_uncertainty_by_repeatability from the aniDom package [30] to estimate the uncertainty in the hierarchy produced using this $30 \%$ subset which produces an $r_{s}$ value. We do this for each interaction category in each group, to assess robustness within each data subset involved in our main analysis of interaction strategies.

Testing for strategic use of interactions in the empirical data: We first apply our method for describing the tendency to interact in relation to rank differences to our empirical dataset of interactions in each of our two vulturine guineafowl social groups, evaluating the three interaction categories independently. Further, 'fights', as defined previously for vulturine guineafowl [33], are omitted from our analysis as they are infrequent and typically occur only between, not within, groups. For each group and interaction category dataset, we run 500 full iterations (i.e. 500 repeats of steps 1-5 in Figure 1). In each of these iterations, the permutation procedure (step 4 in Figure 1 ) is repeated 100,000 times. For each run of the iteration, we fit separate splines for rank differences above and below zero. We do this because interaction strategies towards individuals positioned at relative rank differences of -1 and 1 may differ largely, and by forcing a single spline to pass through zero would under-estimate strategies towards close competitors. We use the R function smooth.spline, with 
parameters: $\mathrm{df}=3$ and lambda $=0.04$, to fit all smoothing splines. All analyses are conducted in the statistical environment $R$ v 3.6.2 [38].

Our analysis investigates the strategy within each interaction category, which does not allow us to evaluate whether individuals preferentially use one type of interaction versus another according to rank differences. For example, both high- and low-cost aggressive interactions might show the same general strategy but to differing extents. Therefore, we employ Bayes' rule to calculate the probability of exhibiting a high-cost aggressive-as opposed to a low-cost aggressive-interaction given the difference in rank. The probability of high-cost aggression $A_{h}$ in an interaction given a rank difference of $R$, is calculated as $P\left(A_{h} \mid R\right)=\frac{P\left(R \mid A_{h}\right) P\left(A_{h}\right)}{P(R)}$. This allows us to evaluate whether there are deviations from the random expression of high-versus low-cost interactions across rank differences. We employ a bootstrapping procedure to estimate the $95 \%$ confidence intervals of $P\left(A_{h} \mid R\right)$.

\section{Results}

\section{Testing the framework}

Our agent-based model shows that in the absence of strategies, simply counting the number of directed interactions among individuals can produce spurious strategic patterns if there is dyadic variation in the opportunity to interact. Specifically, when agents form subgroups assorted by rank, but direct aggressive interactions at random, simply counting directed aggressive interactions results in the appearance of a strategy (Figure 2a). Our approach, which controls for opportunity, correctly reports no strategy (Figure $2 b$ ). Further, when interactions are random, simply counting the number of interactions can give the impression of a 'downwards heuristic' (equal aggression directed towards all lower-ranked individuals [20], Figure 2c) but our method correctly identifies the absence of a strategy (Figure 2d).

\section{Robustness of vulturine guineafowl hierarchies}

In total, we recorded 7358 male-male dominance interactions between $6^{\text {th }}$ September 2019 and $10^{\text {th }}$ February 2021 across two vulturine guineafowl groups. Of these, $25 \%$ were classified as 'high-cost aggressive', $28 \%$ as 'low-cost aggressive' and $46 \%$ as 'submissive' (see Table 2). Hierarchies generated using different interaction categories are highly correlated in both groups (all $r_{s}>0.95$, Figure S1). Additionally, intra-hierarchy repeatability measures are high for all three hierarchies generated in each group (all $r_{s}>0.90$, Table 2), demonstrating low levels of uncertainty within each hierarchy generated in our analyses. 
(a)

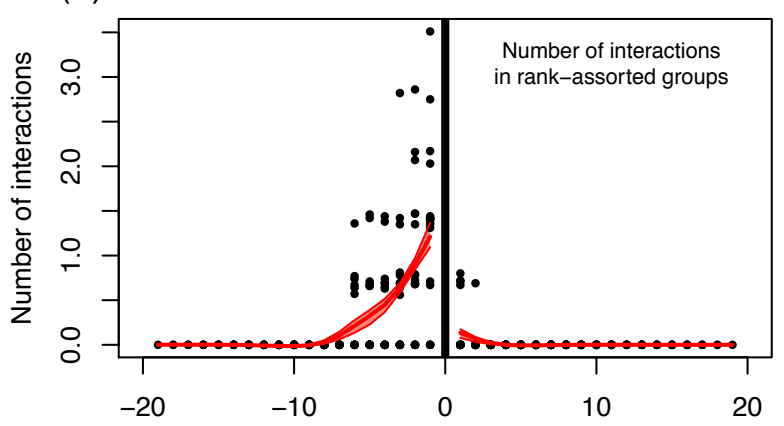

(c)

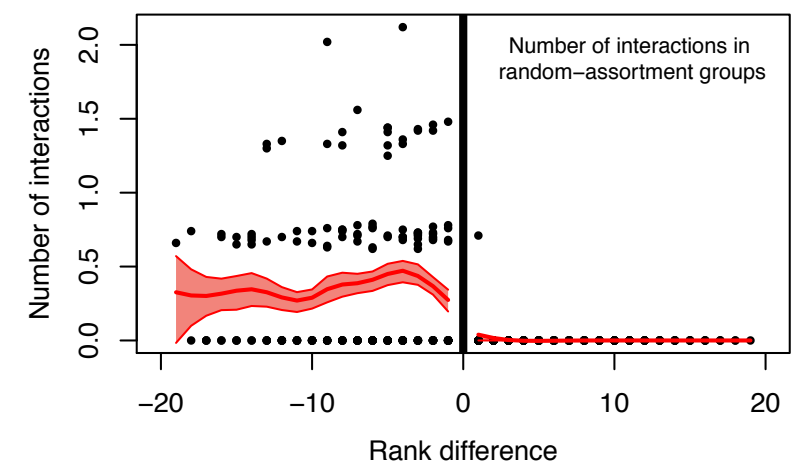

(b)

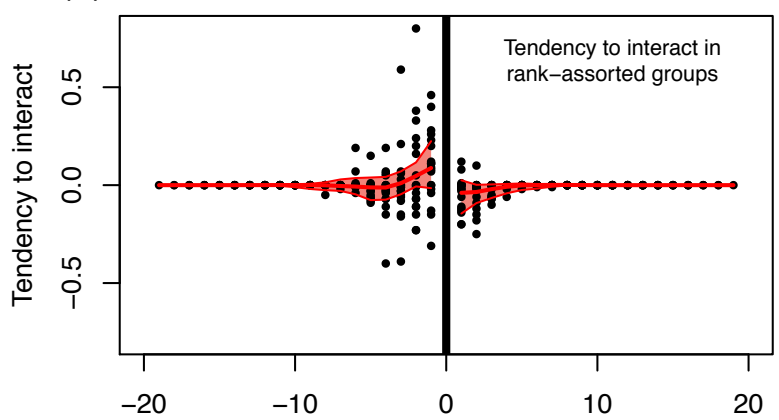

(d)

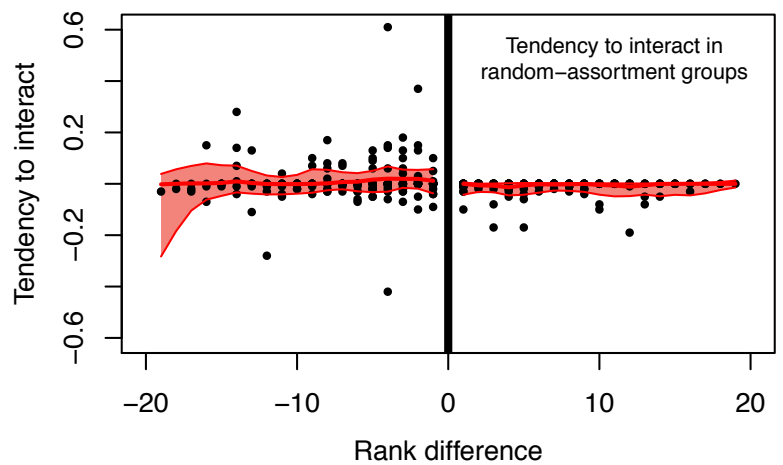

Figure 2. Results from an agent-based model demonstrate that our methodological developments, incorporating null models, account for dyadic opportunity to interact. In simulations where individuals are spatially clustered by rank, but interact without any strategy (i.e. at random), this can give the appearance of a close competitor strategy (a), potentially leading to spurious inference. Controlling for opportunity to interact using a permutation test can correct for this effect (b), with the $95 \%$ range of the analyses (red polygon) overlapping 0 across all values of rank difference. When no rank assortment exists, counts of the number of interactions are high across all negative values of rank difference (c), but interpretation is difficult. Accounting for tendency to interact can confirm that the expression of dominance interactions does not differ from random (d).

Table 2. Data summary and within-category hierarchy repeatability for each study group. The $r_{s}$ value is a Spearman's rank correlation coefficient, estimating within-dataset hierarchy repeatability as calculated using the function estimate_uncertainty_by_repeatability from the aniDom package [30].

\begin{tabular}{cccccc}
\hline Group & Interaction category & no. interactions & Group size & no. interactions per individual & $r_{s}$ value \\
\hline 1 & High-cost aggressive & 1229 & 23 & 53 & 0.96 \\
1 & Low-cost aggressive & 1558 & 23 & 68 & 0.93 \\
1 & Submissive & 2628 & 23 & 114 & 0.95 \\
2 & High-cost aggressive & 627 & 29 & 22 & 0.91 \\
2 & Low-cost aggressive & 529 & 29 & 18 & 0.91 \\
2 & Submissive & 787 & 29 & 27 & 0.93 \\
\hline
\end{tabular}

\section{Interaction strategies}

High-cost aggressive, low-cost aggressive and submissive dominance interactions all show relatively consistent patterns of tendency to interact in relation to the relative positioning of other individuals in the dominance hierarchy (Figure 3). Generally, individuals preferentially direct aggressive interactions towards individuals below themselves in the hierarchy, but direct submissive interactions towards individuals above themselves in the hierarchy (Figure 3). Individuals in both social groups direct significantly more high-cost aggressive interactions than expected by chance towards group 
bioRxiv preprint doi: https://doi.org/10.1101/2021.06.02.446695; this version posted June 2,2021 . The copyright holder for this preprint (which was not certified by peer review) is the author/funder, who has granted bioRxiv a license to display the preprint in perpetuity. It is made available under aCC-BY-NC 4.0 International license.

members occupying ranks immediately below themselves, with the tendency to interact with individuals far away in the hierarchy equal to or less than expected under a random-interactions scenario (Figure 3a,d). In contrast to high-cost aggressive interactions, individuals in both groups do not express low-cost interactions towards their closest competitors more than expected by chance (Figure $3 b, e$ ), but direct low-cost interactions preferentially towards individuals slightly further down the hierarchy (rank difference of $~ 10$ ). At large rank differences the two groups appear to differ, but we note that the number of dyads and, correspondingly, the number of interactions decreases rapidly as rank difference increases (see Figure S2). Submissive interactions are consistently directed towards group members positioned higher in the hierarchy at a tendency to interact equal to or greater than expected at random (Figure 3c,f). These results are consistent when the hierarchy is inferred using Elo scores (standardised to be between 0 and 1 ) instead of ranks (Figure S3).

High-cost aggressive

(a)

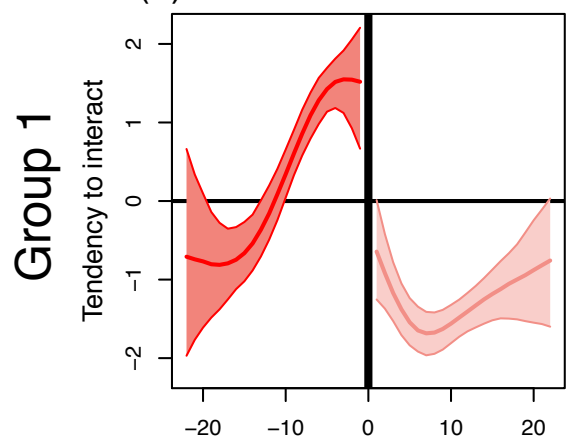

(d)

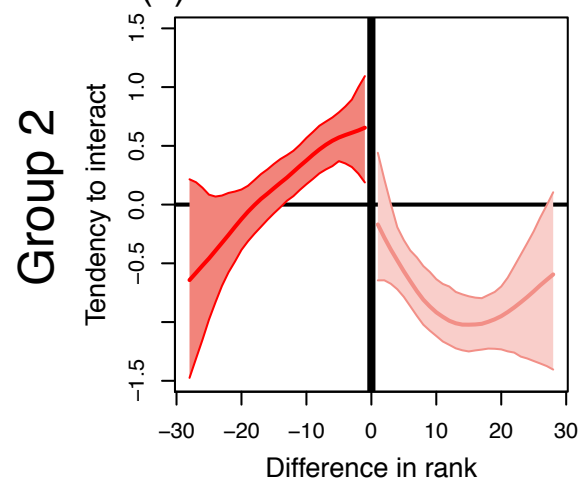

Low-cost aggressive

(b)

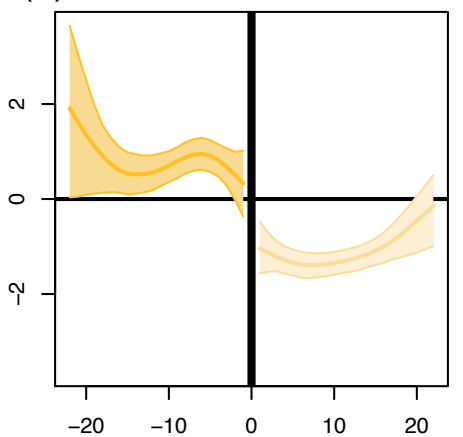

(e)

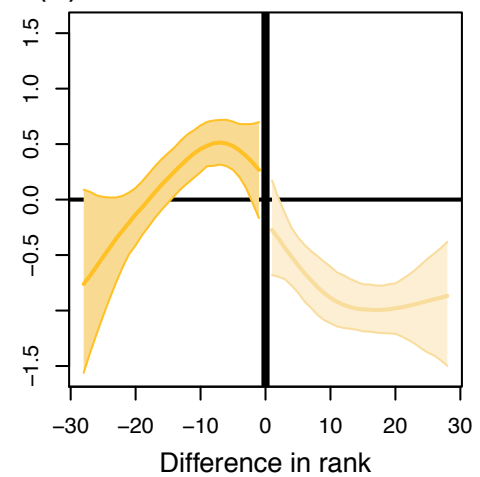

Submissive

(c)

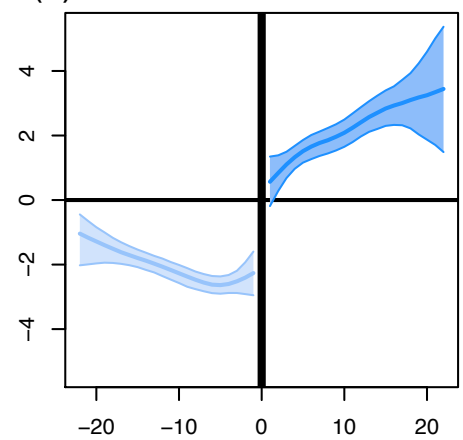

(f)

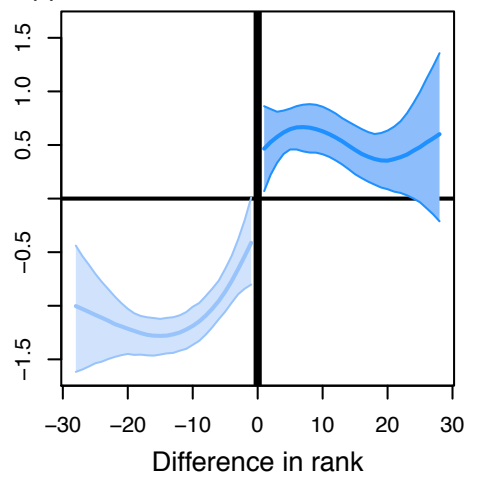

Figure 3. The tendency to interact in relation to relative hierarchy position for three categories of dominance interactions in males of two vulturine guineafowl social groups. Patterns of tendency to interact inferred separately for high-cost aggressive $(\mathbf{a}, \mathbf{d})$, low-cost aggressive $(\mathbf{b}, \mathbf{e})$ and submissive $\mathbf{( c , f )}$ interaction categories for social groups one (a-c) and two (e-f). Each graph shows the median (thick line) tendency to interact and the $95 \%$ range (shaded area) of the estimated tendencies (from the repeated data splitting approach described in Figure 1) plotted against rank difference. A negative difference in rank signifies interactions aimed at lower-ranking individuals, and vice versa for a positive difference in rank. The darker side of each graph relates to aggressive and submissive interactions directed towards lower- and higher-ranking individuals, respectively, and vice versa for the lighter side of the graph. Note that the absolute values of tendency to interact depend on the number of observed interactions and are thus not comparable across graphs. 


\section{Aggression decisions}

While our framework reveals cost-based strategies, it does not allow us to identify the relative allocation of different types of interactions according to cost across rank differences. Using Bayes' rule we find that there appears to be a tendency to disproportionately express high-cost interactions, compared to low-cost interactions, at very small differences in dominance ranks. At least in group one (Figure 4a), individuals express high-cost aggressive interactions towards group members one to three ranks below themselves significantly more than expected by chance. However, in group two, which has much less data-only $41 \%$ of social group one and across more individuals-(see Table 2 ), we detect no such pattern (Figure 4b). Further, the dominant male in group one expressed a surprisingly high number of high-cost interactions towards the most subordinate individual in the group (i.e. at the largest rank difference).

(a)

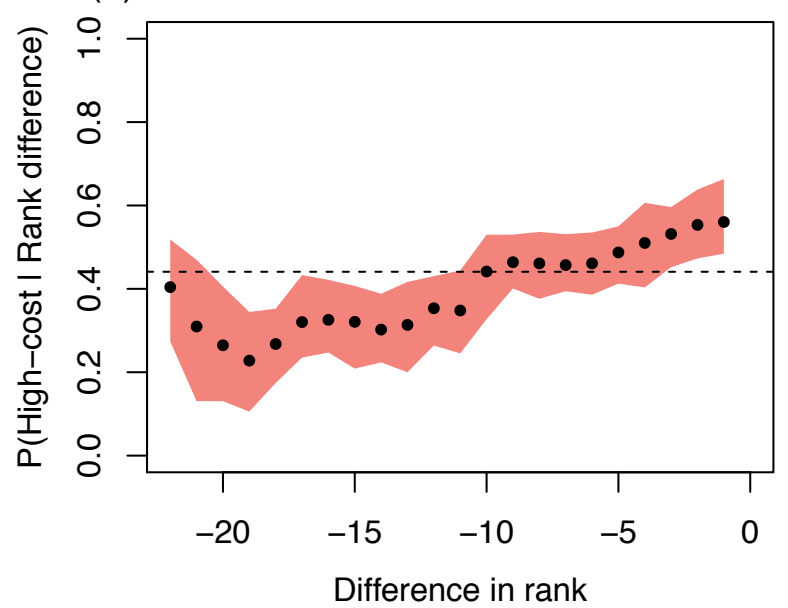

(b)

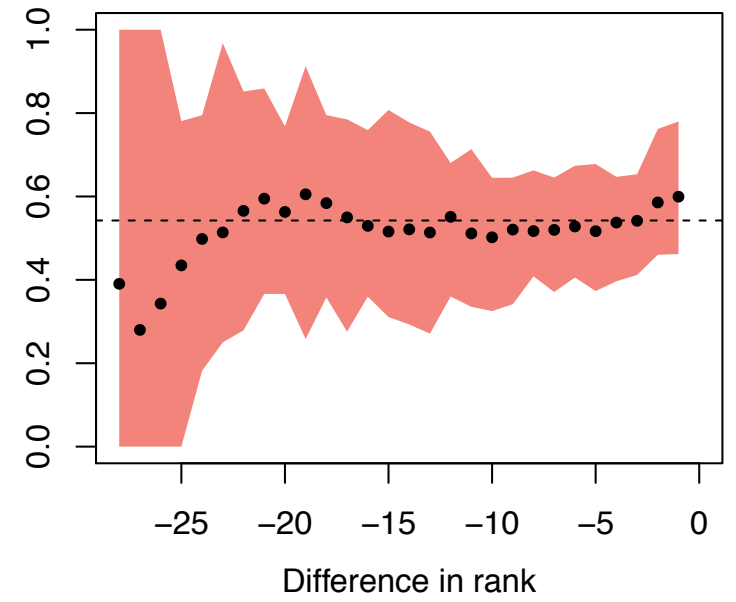

Figure 4. The probability of expressing high- versus low-cost aggressive interactions given rank differences in social groups one (a) and two (b). Black circles represent the observed probability of an individual exhibiting high-cost aggression (versus low-cost aggression) at a given difference in rank. Shaded areas show the $95 \%$ confidence intervals calculated using bootstrapping. The dotted line shows the baseline probability of expressing high-cost interactions versus low-cost interactions in each group (i.e. $P\left(A_{h}\right)$ ).

\section{Discussion}

Recent studies have suggested that rank differences may underlie patterns of aggressive interactions in group-living species $[19,20]$. Here, we extend current methods to incorporate dyadic opportunity to interact and-using an agent-based model-demonstrate that, if unaccounted for, such differences in opportunity can drive patterns that appear to be strategic (i.e. false positives). Applying our novel analytical framework to two groups of vulturine guineafowl-which have extremely well-defined dominance hierarchies-reveals strategic direction of high-cost aggressive interactions towards group members positioned close in the hierarchy. Comparable patterns have been found in previous studies. 
For example, monk parakeets direct aggression towards individuals positioned immediately below themselves at greater frequencies than other group members [19]. Similarly, the frequency of aggressive interactions in both male mountain gorillas [14] and groups of feral dogs (Canis familiaris) [39] is higher among individuals close in rank. We then extend previous findings by revealing that male vulturine guineafowls are more likely to use high-versus low-cost interactions when directing aggression towards very close competitors, at least in one group. Together, our results support the hypothesis that the costs associated with different interaction types can determine dominance interaction strategies.

Our findings rely on the assumption that some interactions are more costly than others. In this case, we expect that costlier interactions should be rarer, making it surprising that high- and low-cost aggressive interactions are present at similar rates in our dataset. One potential explanation is that high-cost interactions are more conspicuous and therefore more detectable to a human observer using an all-occurrence sampling method $[34,40]$. In our dataset, high-cost aggressive interactions are dominated by chases (CHA interaction code), which happen over distances of metres (and several seconds), making them more obvious to a researcher than subtle gapes, displacements or pecks (i.e. GAP, DIS and PEC - low-cost interactions). Our assumed sampling bias is supported by submissive interactions-which are both salient to the observer and should occur most often-being most prevalent in the dataset. An alternative explanation for the high prevalence of interactions in the highcost aggression category is that the costs traditionally associated with high-intensity dominance interactions, such as energy expenditure $[9,10]$, time investment [11] and the risk of injury [12] or predation [13] may not be so considerable in vulturine guineafowl. While quantifying the costs associated with interaction types warrants further research, our methods are not sensitive to the sampling biases discussed above because they always account for their relative frequency in the data.

There are presently two further potential limitations to our framework. First, while we account for the dyadic opportunity to interact, patterns suggesting strategic use of high-cost interactions may be caused by contest escalation among close competitors [41]. In vulturine guineafowl, we do not see typical escalations of contests, and even high-cost contests are short-lived. Fine-scale analysis of video sample data could provide insights into the role of escalation in the dominance interactions of vulturine guineafowl. Second, like most studies of dominance interaction strategies [20], our method assumes the winner of an aggressive interaction is also the initiator. While our method can easily be adapted by directly replacing the winner of the interaction with the initiator, the assumption of initiators being winners is probably reasonable in systems with steep hierarchies. Vulturine guineafowl have one of the steepest hierarchies reported to date, with a rank difference of one corresponding to a $90 \%$ chance of the dominant winning an interaction [33]. Thus, winners are likely to be initiators in 
most interactions (and vice versa for submissive interactions). However, distinguishing 'initiators' from 'winners' will be important in systems with shallow hierarchies or in open populations where individuals may be unfamiliar, as a mismatch could produce spurious findings (though we note that our methods do not generate any patterns under random interactions, see Figure $2 \mathrm{~d}$ ). One final consideration for those applying methods such as ours is to avoid pooling aggressive and submissive interactions, as the winners of aggressive and submissive interactions cannot be equally assumed to be the initiator.

The factors outlined above could reasonably generate apparent differences in strategies across systems. Further, when applying comparative methods across many datasets, Hobson et al. [20] report differences in strategy between groups of the same species. Such findings contrast with the very similar patterns observed across our two groups (different only in low-cost aggressive interactions among dyads with large rank differences and corresponding to low data densities) and in two social groups of monk parakeets [19]. Variation in strategies could appear from differences in the types of resources that individuals compete for [20], or if studies are conducted in different contexts. Alternatively, variation could be driven by methodological differences among studies that are not accounted for in existing analytical approaches, such as what types of interactions are (preferentially) recorded (e.g. whether submissive interactions are included or not) or depending on the setting (e.g. captive vs. wild). An important question is whether we expect that strategies, if adaptive, should meaningfully differ among groups.

It has been suggested that certain interaction strategies demonstrate the use of 'higher-level' or 'global' social information $[15,20]$ as individuals direct interactions in relation to relative rank difference. However, apparently complex strategies may instead be underpinned by simple mechanisms. Hierarchies are often structured by sex and/or age, meaning that preferential interactions with group members of the same class [42] could appear as a close competitor strategy. Patterns resembling close competitor strategies could also arise from individuals directing aggression preferentially towards similar group members in trait-structured hierarchies, for example based on physical size [43] or status badges [44-46]. Alternatively, as stable hierarchies may arise from transitive inference paired with few reference individuals [47], close-competitor or bullying strategies could appear if individuals interact strategically with sets of conspecifics located between pairs of reference individuals in the hierarchy. For example, an individual at rank 10 may have reference individuals at ranks 15 and 20 and may apply one strategy for group members between 10 and 15 in the hierarchy and another for those between 15 and 20. We therefore suggest that caution should be applied when making inferences about the complexity of cognitive processes underlying interaction patterns. Ultimately, given their reduced cognitive demands, simple drivers are more parsimonious 
explanations for strategic interaction patterns in many species, but have not received due consideration.

Our findings add to the growing evidence for group-level dominance interaction strategies across diverse species $[14,19,20]$, and extend our current understanding by demonstrating that individuals may use interactions strategically according to their cost. Yet, there are many further axes of strategies to explore. For example, there may be seasonal variation in the importance of dominance rank-such as when food is scarce or when competition for mates is high-that could modulate strategies. Further, studies of dominance interaction strategies-including ours-thus far consider only grouplevel patterns. Strategies could also vary across individuals, across classes of individuals, or according to individual states. These questions can all be explored by building on the analytical framework developed in our study and others [20]. Finally, while our approach is framed in the context of dominance interactions, it may have a broader applicability; it could readily be applied to any two axes of social interactions, for example how affiliative interactions are directed by measures of similarity or differences among individuals (e.g. kinship).

Acknowledgements. We thank the Mpala Research Centre for logistical support, Kenyan Wildlife Service for authorisation to undertake the research (permit KWS-0016-01-21), the National Commission for Science, Technology and Innovation of Kenya (NACOSTI/P/16/3706/6465) and National Environment Management Authority (NEMA/AGR/68/2017) for permits to Kenyan resources and the National Museums of Kenya for supporting our project. In addition, we thank John Ewoi for assistance in the field as well as Lucy Aplin and the Farine lab for discussions about the study.

Funding. This project was funded by the European Research Council (ERC) under the European Union's Horizon 2020 research and innovation programme (grant agreement number 850859 awarded to DRF), an Eccellenza Professorship Grant of the Swiss National Science Foundation (grant number PCEFP3_187058 awarded to DRF) and the Max Planck Society. TD was supported by the Biotechnology and Biological Sciences Research Council-funded South West Biosciences Doctoral Training Partnership (training grant reference BB/M009122/1). NJB was funded by a Royal Society Dorothy Hodgkin Research Fellowship (DH140080). DP was funded by a DAAD scholarship for post-graduate studies, National Geographic Society Early Career Grant (WW-175ER-17).

Competing interests. The authors declare that no competing interests exist.

Author contributions. DRF, and JP conceived the study. TD, JP, NJB and DRF designed the study. BN, WC and DP collected the data. DRF wrote the initial code and TD analysed the data. TD, NJB and DRF drafted the manuscript and all authors contributed to the final manuscript.

Ethics. Field methods were ethically reviewed by the Max Planck Society Ethikrat Committee (2016_13/1).

Data Accessibility. Data and code to replicate our analyses are available on request. 


\section{References:}

1. Ward A, Webster M. 2016 Sociality: The Behaviour of Group-Living Animals. Switzerland: Springer International Publishing. (doi:10.1007/978-3-319-28585-6)

2. Holekamp KE, Strauss ED. 2016 Aggression and dominance: an interdisciplinary overview. Curr. Opin. Behav. Sci. 12, 44-51. (doi:10.1016/j.cobeha.2016.08.005)

3. Dehnen T, Arbon JJ, Farine DR, Boogert NJ. 2021 How feedback and feed-forward mechanisms link determinants of social dominance. EcoEvoRxiv (doi:10.32942/osf.io/xcp6h)

4. Frank LG. 1986 Social organization of the spotted hyaena Crocuta crocuta. II. Dominance and reproduction. Anim. Behav. 34, 1510-1527. (doi:10.1016/S0003-3472(86)80221-4)

5. Boccia ML, Laudenslager M, Reite M. 1988 Food distribution, dominance, and aggressive behaviors in bonnet macaques. Am. J. Primatol. 16, 123-130. (doi:10.1002/ajp.1350160203)

6. McGowan A, Sharp SP, Simeoni M, Hatchwell BJ. 2006 Competing for position in the communal roosts of long-tailed tits. Anim. Behav. 72, 1035-1043. (doi:10.1016/j.anbehav.2006.02.020)

7. Ellis L. 1995 Dominance and reproductive success among nonhuman animals: a cross-species comparison. Ethol. Sociobiol. 16, 257-333. (doi:10.1016/0162-3095(95)00050-U)

8. Shizuka D, McDonald DB. 2015 The network motif architecture of dominance hierarchies. J. R. Soc. Interface 12, 20150080. (doi:10.1098/rsif.2015.0080)

9. Neat FC, Taylor AC, Huntingford FA. 1998 Proximate costs of fighting in male cichlid fish: the role of injuries and energy metabolism. Anim. Behav. 55, 875-882. (doi:10.1006/anbe.1997.0668)

10. Briffa M, Elwood RW. 2004 Use of energy reserves in fighting hermit crabs. Proc. R. Soc. Lond. B Biol. Sci. 271, 373-379. (doi:10.1098/rspb.2003.2633)

11. Ebensperger LA, Hurtado MJ. 2005 Seasonal changes in the time budget of degus, Octodon degus. Behaviour 142, 91-112. (doi:10.1163/1568539053627703)

12. Clutton-Brock TH, Huchard E. 2013 Social competition and selection in males and females. Philos. Trans. R. Soc. B Biol. Sci. 368, 20130074. (doi:10.1098/rstb.2013.0074)

13. Jakobsson S, Brick O, Kullberg C. 1995 Escalated fighting behaviour incurs increased predation risk. Anim. Behav. 49, 235-239. (doi:10.1016/0003-3472(95)80172-3)

14. Wright E, Galbany J, McFarlin SC, Ndayishimiye E, Stoinski TS, Robbins MM. 2019 Male body size, dominance rank and strategic use of aggression in a group-living mammal. Anim. Behav. 151, 87102. (doi:10.1016/j.anbehav.2019.03.011)

15. Hobson EA. 2020 Differences in social information are critical to understanding aggressive behavior in animal dominance hierarchies. Curr. Opin. Psychol. 33, 209-215. (doi:10.1016/j.copsyc.2019.09.010)

16. Kawakatsu M, Chodrow PS, Eikmeier N, Larremore DB. 2021 Emergence of hierarchy in networked endorsement dynamics. Proc. Natl. Acad. Sci. 118, e2015188118. (doi:10.1073/pnas.2015188118) 
17. Faber DB, Baylis JR. 1993 Effects of body size on agonistic encounters between male jumping spiders (Araneae: Salticidae). Anim. Behav. 45, 289-299. (doi:10.1006/anbe.1993.1033)

18. Hughes M. 1996 Size assessment via a visual signal in snapping shrimp. Behav. Ecol. Sociobiol. 38, 51-57. (doi:10.1007/s002650050216)

19. Hobson EA, DeDeo S. 2015 Social feedback and the emergence of rank in animal society. PLOS Comput. Biol. 11, e1004411. (doi:10.1371/journal.pcbi.1004411)

20. Hobson EA, Mønster D, DeDeo S. 2021 Aggression heuristics underlie animal dominance hierarchies and provide evidence of group-level social information. Proc. Natl. Acad. Sci. 118, e2022912118. (doi:10.1073/pnas.2022912118)

21. Whitehead H. 2008 Analyzing Animal Societies: Quantitative Methods for vertebrate Social Analysis. Chicago: University of Chicago Press.

22. Farine DR, Whitehead H. 2015 Constructing, conducting and interpreting animal social network analysis. J. Anim. Ecol. 84, 1144-1163. (doi:10.1111/1365-2656.12418)

23. Hall CL, Fedigan LM. 1997 Spatial benefits afforded by high rank in white-faced capuchins. Anim. Behav. 53, 1069-1082. (doi:10.1006/anbe.1996.0392)

24. Smith JE, Memenis SK, Holekamp KE. 2007 Rank-related partner choice in the fission-fusion society of the spotted hyena (Crocuta crocuta). Behav. Ecol. Sociobiol. 61, 753-765. (doi:10.1007/s00265-006-0305-y)

25. Hirsch BT. 2011 Within-group spatial position in ring-tailed coatis: balancing predation, feeding competition, and social competition. Behav. Ecol. Sociobiol. 65, 391-399. (doi:10.1007/s00265010-1056-3)

26. Farine DR. 2017 A guide to null models for animal social network analysis. Methods Ecol. Evol. 8, 1309-1320. (doi:10.1111/2041-210X.12772)

27. Schino G, Lasio F. 2019 Mandrills represent their own dominance hierarchy on a cardinal, not ordinal, scale. Anim. Cogn. 22, 1159-1169. (doi:10.1007/s10071-019-01308-8)

28. Levy EJ et al. 2020 A comparison of dominance rank metrics reveals multiple competitive landscapes in an animal society. Proc. R. Soc. B 287, 20201013. (doi:10.1098/rspb.2020.1013)

29. Langley EJG, van Horik JO, Whiteside MA, Madden JR. 2018 Group social rank is associated with performance on a spatial learning task. R. Soc. Open Sci. 5, 171475. (doi:10.1098/rsos.171475)

30. Sánchez-Tójar A, Schroeder J, Farine DR. 2018 A practical guide for inferring reliable dominance hierarchies and estimating their uncertainty. J. Anim. Ecol. 87, 594-608. (doi:10.1111/13652656.12776)

31. Papageorgiou D, Christensen C, Gall GEC, Klarevas-Irby JA, Nyaguthii B, Couzin ID, Farine DR. 2019 The multilevel society of a small-brained bird. Curr. Biol. 29, R1120-R1121. (doi:10.1016/j.cub.2019.09.072)

32. Papageorgiou D, Farine DR. 2020 Group size and composition influence collective movement in a highly social terrestrial bird. eLife 9, e59902. (doi:10.7554/eLife.59902) 
33. Papageorgiou D, Farine DR. 2020 Shared decision-making allows subordinates to lead when dominants monopolize resources. Sci. Adv. 6, eaba5881. (doi:10.1126/sciadv.aba5881)

34. Altmann J. 1974 Observational study of behavior: sampling methods. Behaviour 49, 227-266. (doi:10.1163/156853974X00534)

35. Newton-Fisher NE. 2017 Modeling social dominance: Elo-ratings, prior history, and the intensity of aggression. Int. J. Primatol. 38, 427-447. (doi:10.1007/s10764-017-9952-2)

36. Noë R, de Waal FBM, van Hooff JARAM. 1980 Types of dominance in a chimpanzee colony. Folia Primatol. (Basel) 34, 90-110. (doi:10.1159/000155949)

37. Hayaki H, Huffman MA, Nishida T. 1989 Dominance among male chimpanzees in the Mahale Mountains National Park, Tanzania: A preliminary study. Primates 30, 187-197. (doi:10.1007/BF02381303)

38. R Core Team. 2019 R: A language and environment for statistical computing. Vienna, Austria: $\mathrm{R}$ Foundation for Statistical Computing. See https://www.R-project.org/.

39. Silk MJ, Cant MA, Cafazzo S, Natoli E, McDonald RA. 2019 Elevated aggression is associated with uncertainty in a network of dog dominance interactions. Proc. R. Soc. B Biol. Sci. 286, 20190536. (doi:10.1098/rspb.2019.0536)

40. Bernstein IS. 1991 An empirical comparison of focal and ad libitum scoring with commentary on instantaneous scans, all occurrence and one-zero techniques. Anim. Behav. 42, 721-728. (doi:10.1016/S0003-3472(05)80118-6)

41. Parker GA. 1974 Assessment strategy and the evolution of fighting behaviour. J. Theor. Biol. 47, 223-243. (doi:10.1016/0022-5193(74)90111-8)

42. Aureli F, Schino G. 2019 Social complexity from within: how individuals experience the structure and organization of their groups. Behav. Ecol. Sociobiol. 73, 6. (doi:10.1007/s00265-018-2604-5)

43. Nakano S. 1994 Variation in agonistic encounters in a dominance hierarchy of freely interacting red-spotted masu salmon (Oncorhynchus masou ishikawai). Ecol. Freshw. Fish 3, 153-158. (doi:10.1111/j.1600-0633.1994.tb00017.x)

44. Thompson CW, Moore MC. 1991 Throat colour reliably signals status in male tree lizards, Urosaurus ornatus. Anim. Behav. 42, 745-753. (doi:10.1016/S0003-3472(05)80120-4)

45. Setchell JM, Wickings EJ. 2005 Dominance, status signals and coloration in male mandrills (Mandrillus sphinx). Ethology 111, 25-50. (doi:10.1111/j.1439-0310.2004.01054.x)

46. Santos ESA, Scheck D, Nakagawa S. 2011 Dominance and plumage traits: meta-analysis and metaregression analysis. Anim. Behav. 82, 3-19. (doi:10.1016/j.anbehav.2011.03.022)

47. Doi K, Nakamaru M. 2021 Heuristics facilitates the evolution of transitive inference and social hierarchy in a large group. ArXiv , 2103.14363. 


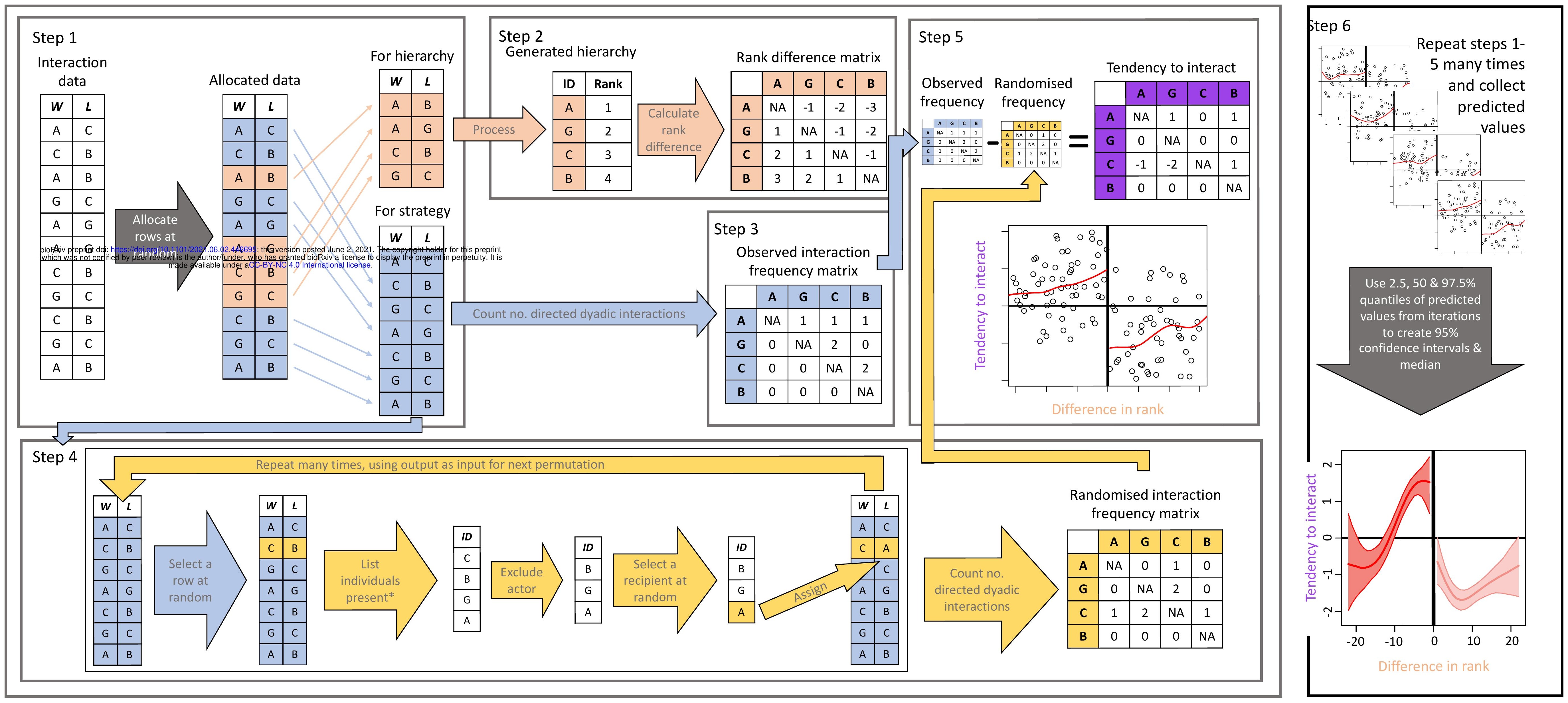


(a)

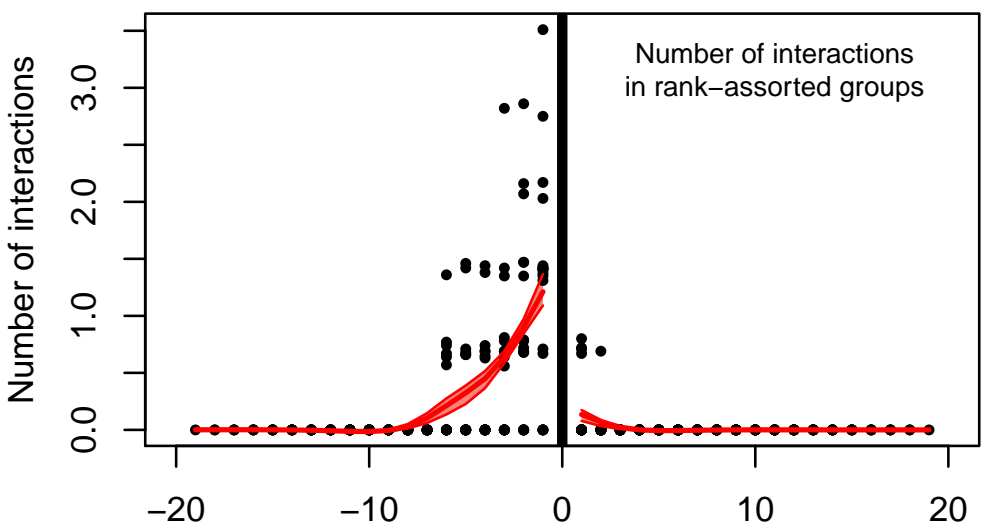

(c)

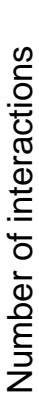

2

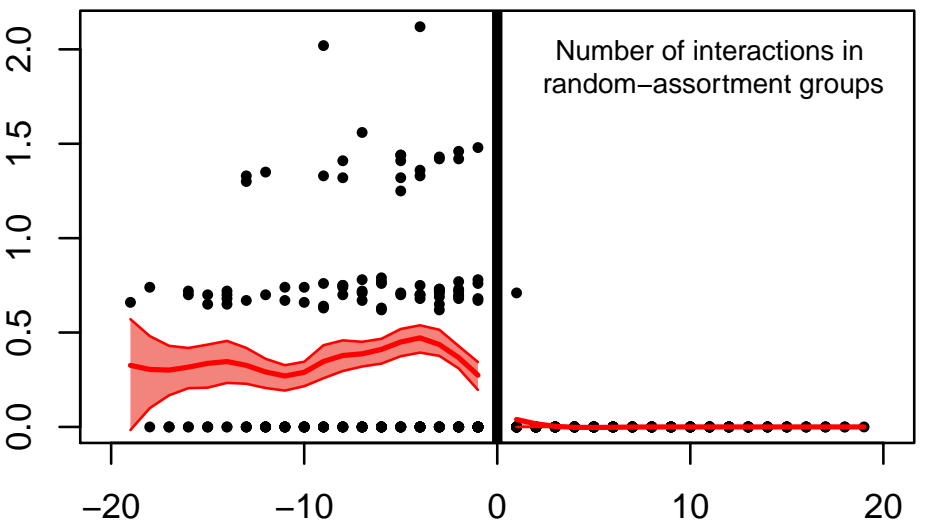

Rank difference 
(a)

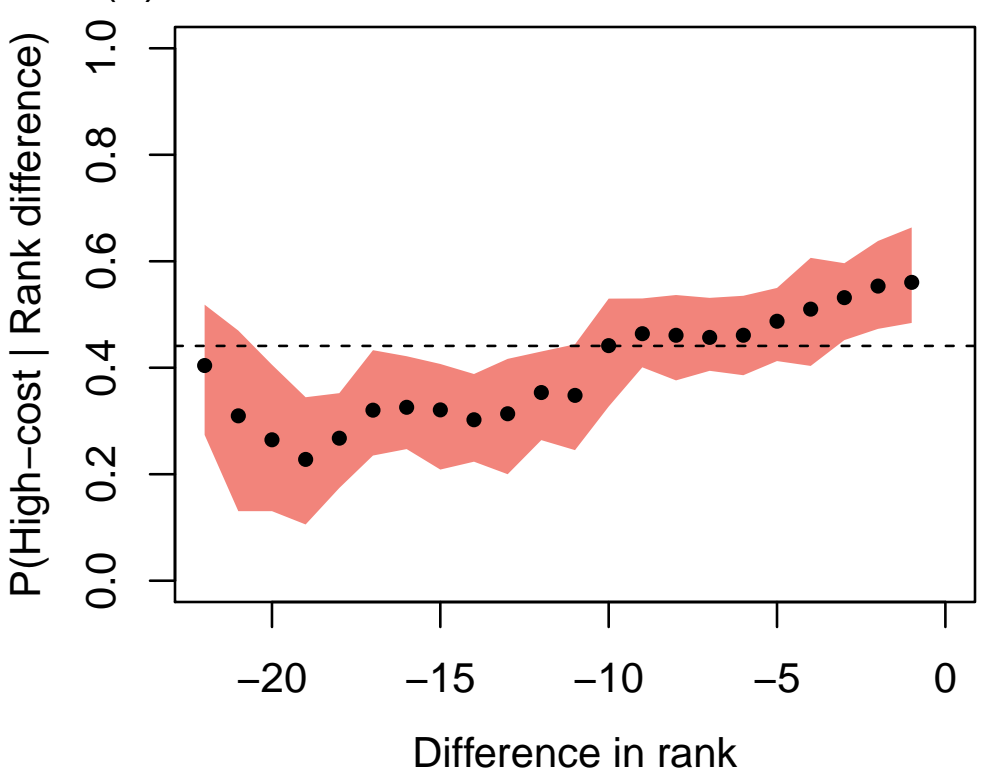

(b)

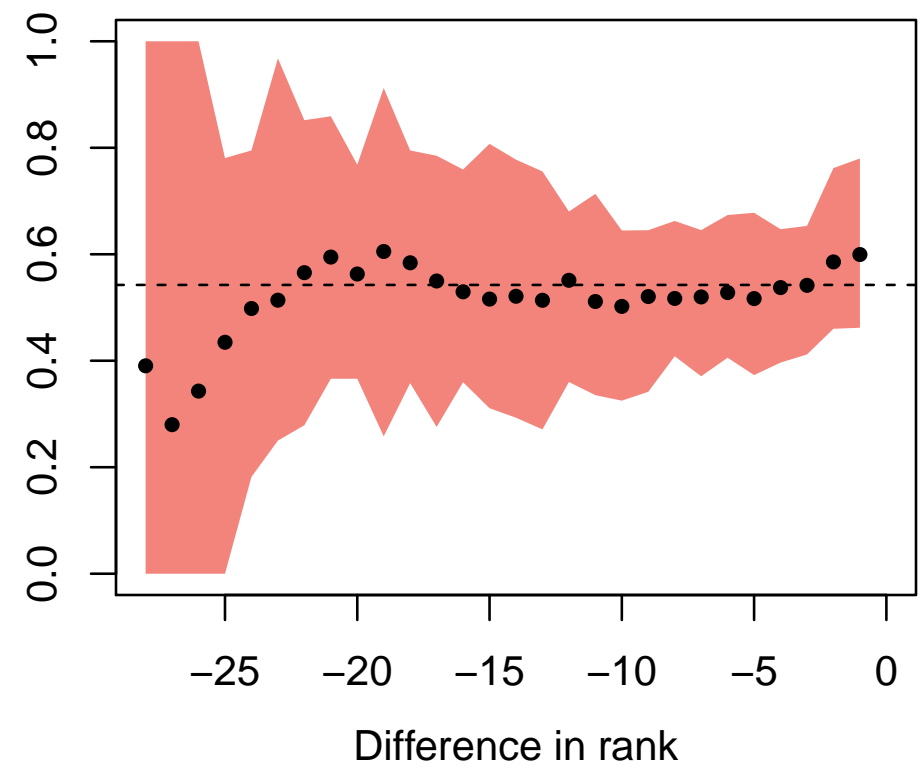

